\title{
POSITIVE MARTINGALES AND THEIR INDUCED MEASURES
}

\author{
BENNETT EISENBERG AND GAN SHIXIN
}

\begin{abstract}
Conditions for the absolute continuity of probability measures are given in terms of limits of sequences of Radon-Nikodym derivatives. In the other direction, conditions for the optional stopping theorem for positive martingales are given in terms of properties of their induced measures.
\end{abstract}

Introduction. Let $(\Omega, \mathcal{E}, P)$ and $(\Omega, \mathcal{E}, Q)$ be probability spaces and let $\left(\widetilde{\mathscr{F}}_{n}, n \in N\right)$ be an increasing sequence of sub- $\sigma$-fields of $\mathcal{E}$, where $N$ is the set of nonnegative integers. If $Q$ is absolutely continuous with respect to $P$ over $\mathscr{F}_{n}$ we write $Q_{n} \ll P_{n}$ and $d Q_{n}=f_{n} d P_{n}$, where $f_{n}$ is the Radon-Nikodym derivative over $\mathscr{F}_{n} \cdot\left(f_{n}, n \in N\right)$ is then a positive martingale with respect to $\left(\mathscr{F}_{n}, n \in N\right)$ and $P$. Hence $\lim f_{n}$ exists a.s. $P$. Conversely, if $\left(f_{n}, n \in N\right)$ is a positive martingale with respect to $\left(\mathscr{F}_{n}, n \in N\right)$ and $P$, then there is a well-defined finitely additive set function $Q$ on $\cup_{n=0}^{\infty} \mathscr{F}_{n}$ with $Q(A)=\int_{A} f_{n} d P$ for $A$ in $\mathscr{F}_{n} . Q$ is called the measure induced by $\left(f_{n}, n \in N\right)$. Set $\widetilde{F}_{\infty}=\sigma\left(\cup_{n=0}^{\infty} \mathscr{F}_{n}\right)$.

The following theorem brings together several well-known results on the convergence of positive martingales (see Ash $[1972, \S 7.6]$ and Neveu [1975, Proposition III-1-1]) and indicates the general significance of the problem considered in this paper. All positive martingales in this paper are normalized.

General Theorem. Assume $f_{n}$ is a positive martingale with respect to $\left(\mathscr{F}_{n}, n \in N\right)$ and $P$, and $Q$ is the induced measure. Then the following are equivalent:

(i) $\int \lim f_{n} d P=1$.

(ii) $f_{n} \rightarrow \lim f_{n}$ in $L^{1}(P)$.

(iii) $\left(f_{n}, n \in N\right)$ is uniformly integrable $(P)$.

(iv) There exists an $f$ with $\mathcal{E}_{p}\left(f \mid F_{n}\right)=f_{n}$ for $n$ in $N$.

(v) $f_{k}=\mathcal{E}_{p}\left(\lim f_{n} \mid \mathscr{F}_{k}\right)$ for $k$ in $N$.

(vi) $Q$ extends to a $\sigma$-additive measure $Q_{\infty}$ on $\widetilde{F}_{\infty}$ and $Q_{\infty} \ll P_{\infty}$.

(vii) $Q$ extends to $Q_{\infty}$ and $d Q_{\infty}=\lim f_{n} d P_{\infty}$.

In a recent paper Kabanov, Lipcer and Shiryaev (hereafter K.L.S.) have given another necessary and sufficient condition for $Q_{\infty} \ll P_{\infty}$ and have shown its usefulness. The result (K.L.S. [1977, Lemma 6]) states that $Q_{\infty} \ll P_{\infty}$ if and only if $Q\left(\lim f_{n}=\infty\right)=0$. They also show that two probability measures $Q$ and $P$ with $Q_{n} \ll P_{n}$ for $n$ in $N$ are singular over $\mathscr{F}_{\infty}$ if and only if $Q\left(\lim f_{n}=\infty\right)=1$.

Received by the editors January 10, 1982.

1980 Mathematics Subject Classification. Primary 60G30; Secondary 60G45. 
$\$ 1$ contains a simple proof of an extension of the results of K.L.S. The proof avoids the technical problems associated with dividing by zero in the K.L.S. approach. $\$ 2$ gives some properties of likelihood ratios or extended Radon-Nikodym derivatives. $\$ 3$ includes conditions for the absolute continuity and singularity over $\mathscr{F}_{\nu}$, the $\sigma$-field of events prior to a stopping time $\nu$. The proofs show how useful the likelihood ratio can be. Moreover, the results lead to necessary and sufficient conditions for the optional stopping theorem of the same type as the K.L.S. conditions for absolute continuity.

1. Absolute continuity over $\mathscr{F}_{\infty}$. Let $R=\frac{1}{2}(P+Q)$. Then $Q \ll R$ over a general $\sigma$-field $\mathscr{F} \subset \mathcal{E}$. If $d Q=y d R$ then $Q(A)=\frac{1}{2} \int_{A} y d Q+\frac{1}{2} \int_{A} y d P$, so $\int_{A}(2-y) d Q=$ $\int_{A} y d P$ for all $A$ in $\mathscr{F}$. In particular, by first letting $A=\{y=2\}$ and then $A=\{y=$ $0\}$, we see that $Q(y=0)=P(y=2)=0$.

LEMMA 1. (a) $Q \ll P$ if and only if $Q(y=2)=0$.

(b) The following are equivalent: (i) $Q \perp P$, (ii) $Q(y=2)=1$, (iii) $P(y=0)=1$.

Proof. (a) Assume $Q(y=2)=0$ and $P(A)=0$. Then $\int_{A}(2-y) d Q=\int_{A} y d P=$ 0 . But $Q(2-y>0)=1$. Hence $Q(A)=0$. This shows $Q \ll P$.

Conversely, assume $Q \ll P$. Then since $P(y=2)=0$ we must have $Q(y=2)=0$.

(b) (i) $\Leftrightarrow$ (ii). Assume $Q(y=2)=1$. Then since $P(y=2)=0$ we have $Q \perp P$.

Conversely, let $P(A)=0$ and $Q(A)=1$ for some $A$. Then $\int_{A}(2-y) d Q=\int_{A} y d P$ $=0$. Hence $Q(2-y \neq 0)=0$. That is, $Q(y=2)=1$.

The proof of (i) $\Leftrightarrow$ (iii) is similar.

An extension of the K.L.S. results is an immediate consequence.

THEOREM 1. Assume $d Q_{n}=f_{n} d P_{n}$ and $Q$ extends to a $\sigma$-additive measure on $\mathscr{F}_{\infty}$.

(a) The following are equivalent: (i) $Q_{\infty} \ll P_{\infty}$, (ii) $Q\left(\lim f_{n}=\infty\right)=0$, (iii) $Q\left(\lim \sup f_{n}=\infty\right)=0$.

(b) The following are equivalent: (i) $Q_{\infty} \perp P_{\infty}$, (ii) $Q\left(\lim f_{n}=\infty\right)=1$, (iii) $Q\left(\lim \sup f_{n}=\infty\right)=1$, (iv) $P\left(\lim f_{n}=0\right)=1$.

Proof. $d Q_{n}=f_{n} d P_{n}$ and $d R_{n}=\frac{1}{2}\left(1+f_{n}\right) d P_{n}$. Thus $d Q_{n}=2 f_{n}\left(1+f_{n}\right)^{-1} d R_{n}$, and and by the General Theorem $2 f_{n}\left(1+f_{n}\right)^{-1}$ converges a.s. $R$ to $y_{\infty}$, where $d Q_{\infty}=y_{\infty} d R_{\infty}$. In particular, $\lim 2 f_{n}\left(1+f_{n}\right)^{-1}=y_{\infty}$ a.s. $Q$.

(a) and (b) (i) $\Leftrightarrow$ (ii). $Q\left(y_{\infty}=2\right)=Q\left(\lim 2 f_{n}\left(1+f_{n}\right)^{-1}=2\right)=Q\left(\lim f_{n}=\infty\right)$. Now apply Lemma 1.

(b) (ii) $\Leftrightarrow$ (iii). $Q\left(\lim 2 f_{n}\left(1+f_{n}\right)^{-1}\right.$ exists) $=1$.

(i) $\Leftrightarrow$ (iv). $\quad P\left(y_{\infty}=0\right)=P\left(\lim 2 f_{n}\left(1+f_{n}\right)^{-1}=0\right)=P\left(\lim f_{n}=0\right)$. Now apply Lemma 1.

The condition that $Q$ extend to a $\sigma$-additive measure on $\mathscr{F}_{\infty}$ is very mild. It is always the case in the standard situation where $\Omega=R^{\infty},\left(X_{n}, n \in N\right)$ are the coordinate functions, $\mathscr{F}_{n}=\sigma\left(X_{0}, \ldots, X_{n}\right)$, and $f_{n}$ is a function of $\left(X_{1}, \ldots, X_{n}\right)$. This follows from the Kolmogoroff existence theorem. In this case we say $\left(f_{n}, n \in N\right)$ is a standard positive martingale. 
2. Likelihood ratios. The Lebesgue decomposition also follows from the identity $\int_{A}(2-y) d Q=\int_{A} y d P$. Let $h=y(2-y)^{-1}$, where this expression is defined as $\infty$ for $y=2$.

Lemma 2. (a) $Q(A)=\int_{A} h d P+Q(A \cap\{h=\infty\})$, where $P(h=\infty)=0$.

(b) The following are equivalent: (i) $Q \ll P$, (ii) $Q(h=\infty)=0$, (iii) $\int h d P=1$.

(c) The following are equivalent: (i) $Q \perp P$, (ii) $Q(h=\infty)=1$, (iii) $\int h d P=0$.

Proof. (a)

$$
Q(A \cap\{h \neq \infty\})=\int_{A \cap\{y \neq 2\}} \frac{2-y}{2-y} d Q=\int_{A \cap\{y \neq 2\}} \frac{y}{2-y} d P=\int_{A} h d P
$$

since $\{y=2\}=\{h=\infty\}$ and $P(y=2)=0$ always.

(b) and (c) (i) $\Leftrightarrow$ (ii). From Lemma 1 .

(b) and (c) (ii) $\Leftrightarrow$ (iii). Since $1=Q(\Omega)=\int h d P+Q(h=\infty)$.

$h$ is an extended Radon-Nikodym derivative called the likelihood ratio in statistics. Let $h_{n}$ be the likelihood ratio over $\mathscr{F}_{n}$. We have

LemMa 3. $h_{n} \rightarrow h_{\infty}$ a.s. $P+Q$.

Proof. Let $d Q_{n}=y_{n} d R_{n}$. Then $y_{n} \rightarrow y_{\infty}$ a.s. $R$. Hence $h_{n}=y_{n}\left(2-y_{n}\right)^{-1} \rightarrow$ $y_{\infty}\left(2-y_{\infty}\right)^{-1}=h_{\infty}$ a.s. $R$.

This lemma extends K.L.S. [1977, Lemma 5], which assumes $Q_{n} \ll P_{n}$ for $n$ in $N$. It shows that in a certain sense likelihood ratios are easier to work with than Radon-Nikodym derivatives since limits of likelihood ratios are always likelihood ratios.

Corollary. Let $P_{\infty}$ and $Q_{\infty}$ be probability measures on $\widetilde{F}_{\infty} \cdot Q_{\infty} \perp P_{\infty}$ if and only if $Q\left(\lim h_{n}=\infty\right)=1$.

Proof. $Q_{\infty} \perp P_{\infty} \Leftrightarrow Q\left(h_{\infty}=\infty\right)=1 \Leftrightarrow Q\left(\lim h_{n}=\infty\right)=1$.

This corollary does not require $Q_{n} \ll P_{n}$ for $n$ in $N$ as in the previous results.

3. Absolute continuity over $\mathscr{F}_{\nu}$. Let $\bar{N}=N \cup\{\infty\}$. $\nu$ is called a stopping time with respect to $\left\{\mathscr{F}_{n}, n \in \bar{N}\right\}$ if $\{\nu=n\} \in \mathscr{F}_{n}$ for $n$ in $\bar{N}$. $\mathscr{F}_{\nu}=\left\{A \mid A \cap\{\nu=n\} \in \mathscr{F}_{n}\right.$ for $n$ in $\bar{N}\}$ is the $\sigma$-field of events up to time $\nu$. Over the $\sigma$-field $\mathscr{F}_{\nu}$ we write $d Q_{\nu}=y_{\nu} d R_{\nu}$.

LEMMA 4. Assume $P$ and $Q$ are probability measures on $\mathscr{F}_{\infty}$.

(a) $y_{\nu}=y_{n}$ on $\{\nu=n\}$ for $n$ in $\bar{N}$.

(b) $h_{\nu}=h_{n}$ on $\{\nu=n\}$ for $n$ in $\bar{N}$.

Proof. (a) If $A$ is in $\mathscr{F}_{\nu}$ then $A=\cup_{n \in \bar{N}}\left(A_{n} \cap\{\nu=n\}\right)$ for $A_{n}$ in $\mathscr{F}_{n}$. But $Q\left(A_{n} \cap\{\nu=n\}\right)=\int_{A_{n} \cap\{\nu=n\}} y_{n} d R$ since $A_{n} \cap\{\nu=n\} \in \mathscr{F}_{n}$. Thus $y_{\nu}=y_{n}$ on $\{\nu=$ $n\}$ by the uniqueness of the Radon-Nikodym derivative.

(b) $h_{\nu}=y_{\nu}\left(2-y_{\nu}\right)^{-1}$.

COROLlaRY. (a) The following are equivalent:

(i) $Q_{\nu} \ll P_{\nu}$.

(ii) $Q\left(h_{\nu}=\infty\right)=0$.

(iii) $Q\left(\nu=n, h_{n}=\infty\right)=0$ for $n$ in $\bar{N}$. 
(iv) $\int h_{\nu} d P=1$.

(b) The following are equivalent:

(i) $Q_{\nu} \perp P_{\nu}$.

(ii) $Q\left(h_{\nu}=\infty\right)=1$.

(iii) $Q\left(\nu=n, h_{n}<\infty\right)=0$ for $n$ in $\bar{N}$.

(iv) $\int h_{\nu} d P=0$.

Proof. (a) and (b) (i) $\Leftrightarrow$ (ii) $\Leftrightarrow$ (iv) follows by Lemma 2, and (ii) $\Leftrightarrow$ (iii) follows from Lemma 4.

TheOREM 2. Assume $Q$ and $P$ are probability measures. Assume $d Q_{n}=f_{n} d P_{n}$ for $n$ in $N$. Then:

(a) $Q_{\nu} \ll P_{\nu}$ if and only if $Q\left(\lim f_{n}=\infty, \nu=\infty\right)=0$,

(b) $Q_{\nu} \perp P_{\nu}$ if and only if $Q\left(\lim f_{n}=\infty, \nu=\infty\right)=1$.

Proof. $h_{n}=f_{n}<\infty$ for $n$ in $N$ and $h_{\infty}=\lim f_{n}$. Now apply the Corollary to Lemma 4.

CoRollaRY 1. $d Q_{n}=f_{n} d P_{n}$ for $n$ in $N$ and $Q(\nu=\infty)=0$ then $Q_{\nu} \ll P_{\nu}$.

Corollary 2. If $d Q_{n}=f_{n} d P_{n}$ for $n$ in $N$ and $Q_{\infty} \perp P_{\infty}$, then:

(a) $Q_{\nu} \ll P_{\nu}$ if and only if $Q(\nu=\infty)=0$,

(b) $Q_{\nu} \perp P_{\nu}$ if and only if $Q(\nu=\infty)=1$.

Proof. If $Q_{\infty} \perp P_{\infty}$ then $Q\left(\lim f_{n}=\infty\right)=1$ by Theorem 1 .

It also follows from the Lebesgue decomposition that when $Q_{\nu} \ll P_{\nu}$ we have $d Q_{\nu}=f_{\nu} d P$, where $f_{\nu}=f_{n}$ for $n$ in $N$ and $f_{\nu}=\lim f_{n}$ on $\{\nu=\infty\}$.

These results have an interesting interpretation in terms of the optional stopping problem. The next theorem gives necessary and sufficient conditions for two versions of the optional stopping theorem for positive martingales that are analogous to the K.L.S. necessary and sufficient conditions for absolute continuity.

THEOREM 3. Assume $\left(f_{n}, n\right.$ in $\left.N\right)$ is a positive martingale and the induced measure $Q$ is $\sigma$-additive. Then:

(a) $1=\int_{\{\nu<\infty\}} f_{\nu} d P+\int_{\{\nu=\infty\}} \lim f_{n} d P+Q\left(\nu=\infty, \lim f_{n}=\infty\right)$.

(b) $\int f_{\nu} d P=1 \Leftrightarrow Q\left(\nu=\infty, \lim f_{n}=\infty\right)=0$.

(c) The following are equivalent:

(i) $1=\int_{\{\nu<\infty\}} f_{\nu} d P$,

(ii) $Q\left(\nu=\infty, \lim f_{n}=\infty\right)=0$ and $P\left(\lim f_{n}>0, \nu=\infty\right)=0$,

(iii) $Q(\nu=\infty)=0$.

Proof. (a) follows from the Lebesgue decomposition in Lemma 2 since $h_{\nu}=f_{\nu}$ and $\left\{h_{\nu}=\infty\right\}=\left\{\lim f_{n}=\infty, \nu=\infty\right\}$.

(b) and (c) (i) $\Leftrightarrow$ (ii) follows from (a).

(c) (ii) $\Leftrightarrow$ (iii) again follows from Lemma 2 since

$$
Q(\nu=\infty)=\int_{\{\nu=\infty\}} f_{\nu} d P+Q\left(\nu=\infty, f_{\nu}=\infty\right) .
$$


The condition that $Q$ is $\sigma$-additive can, of course, be replaced by the condition that $\left(f_{n}, n\right.$ in $\left.N\right)$ is a standard positive martingale.

In the general theory of martingales $\nu$ is called a regular stopping time if $f_{\nu \wedge n}$ converges to $f_{\nu}$ in $L^{\prime}(P)$. It follows from Scheffe's lemma that this is the case if $\int f_{\nu} d P=1$. Thus Theorem 3 also gives necessary and sufficient conditions for $\nu$ to be regular.

The final corollary, which is an immediate consequence of the theorem, shows the usefulness of the conditions in terms of $Q$.

COROLlaRY. Under the conditions of Theorem 3, let $\nu_{1}$ and $\nu_{2}$ be stopping times with $\left\{\nu_{1}=\infty\right\} \subset\left\{\nu_{2}=\infty\right\}$. Then:

(a) $\int f_{\nu_{2}} d P=1 \Rightarrow \int f_{\nu_{1}} d P=1$,

(b) $\int_{\left\{\nu_{2}<\infty\right\}} f_{\nu_{2}} d P=1 \Rightarrow \int_{\left\{\nu_{1}<\infty\right\}} f_{\nu_{1}} d P=1$.

\section{REFERENCES}

[1972] Robert B. Ash, Real analysis and probability, Academic Press, New York.

[1977] J. M. Kabanov, R. S. Lipcer and A. N. Shiryaev, On the question of absolute continuity and singularity of probability measures, Math. USSR Sb. 33, 203-221.

[1975] J. Neveu, Discrete parameter martingales, North-Holland, Amsterdam.

Department of Mathematics, Lehigh University, Bethlehem, Pennsylvania 18015 (Current address of Bennett Eisenberg)

Current address (Gan Shixin): Department of Mathematics, Wuhan University, Wuchang, China 